\title{
Percutaneous Computer Assisted Iliosacral Screwing: Clinical Validation
}

\author{
Lionel Carrat ${ }^{\mathrm{a}}$, Jérome Tonetti ${ }^{\mathrm{b}}$, M.D, \\ Philippe Merloz ${ }^{\mathrm{c}}$, M.D, Jocelyne Troccaz ${ }^{\mathrm{a}}$, Ph.D. \\ e-mail : lionel.carrat@imag.fr
}

\author{
${ }^{a}$ TIMC Laboratory, Faculté de médecine, I.A.B., 38706 La Tronche (France) \\ ${ }^{\mathrm{b}}$ Anatomy Laboratory, Pr Chirossel, University Joseph Fourier 38043 Grenoble \\ c Orthopaedic Department, CHU A. Michallon - BP 217 - 38043 Grenoble
}

\begin{abstract}
This paper describes the clinical validation of an image-guided system for the percutaneous placement of iliosacral screws. The goals of the approach are to decrease surgical complications, with a percutaneous technique, and to increase the accuracy and security of screw positioning thanks to a computer assisted system. Pre-operative planning is performed on CT-scan images and a 3D model is built. During surgery, tools are tracked with an optical localizer. An ultrasound acquisition is performed and images are segmented to obtain 3D intra-operative data that are registered with the CT-scan 3D model. The surgeon is assisted during drilling and screwing processes with re-sliced CT-scan images displayed on the computer screen and comparison between pre-operative planning and tools position. The system was validated in a cadaver study ${ }^{[1]}$. The clinical validation has then started and four patients have been successfully instrumented.
\end{abstract}

Keywords : orthopaedics, clinical results, ultrasound-based registration.

\section{Introduction}

Unstable pelvic ring fractures with complete posterior ring disruption (see Fig. 1A) require a surgical fixation as described in ${ }^{[2]}$. The non-operative treatment is not adequate to stabilize the closed reduction in order to prevent hemipelvic ascension or pseudarthrosis of the fracture. The surgical procedure consists in drilling two holes and introducing two screws through the iliosacral joint into the sacral ala and the body of the first sacral vertebra (see Fig. 1B). The trajectory of the drill must avoid the close anatomic elements ${ }^{[3]}$ that are the first sacral nerve root in the S1-S2 foramen, the lumbosacral nerve trunc, the roots of the cauda equina, the iliac vessels and the peritonial cavity.

Two surgical procedures are currently used.

The first one is open reduction described by several authors ${ }^{[4,5,6]}$. In order to secure the procedure, empirical reference marks must be taken. As a consequence, a large surgical exposure of the sacrum and the posterior part of the fossa iliaca lateral must be done. Complications like blood loss, hematoma and wound infection are not rare after such exposure because of the perineum's proximity. 

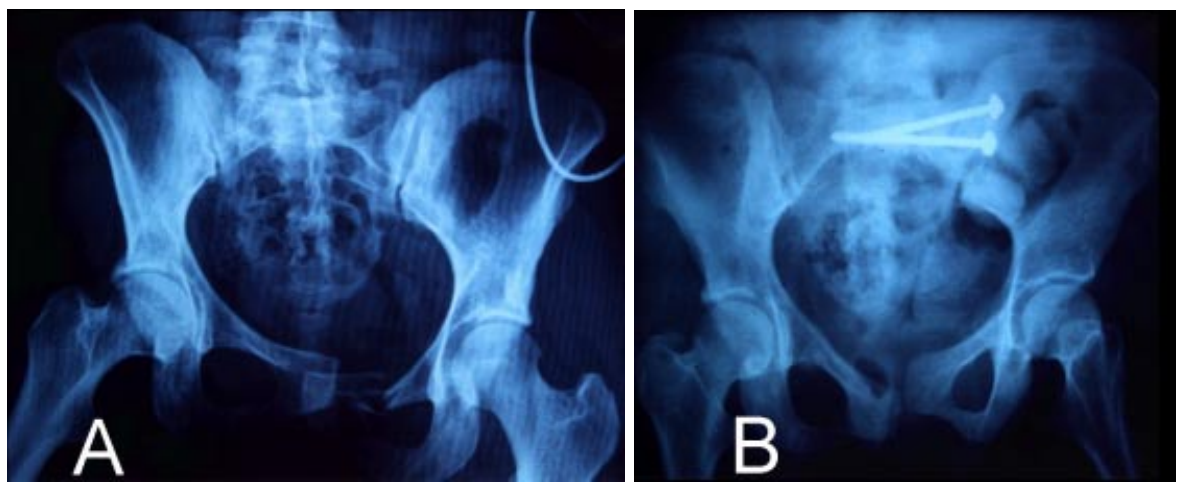

Fig. 1 : (A) Unstable pelvic ring fracture with complete disruption of the posterior arch. (B) Example of iliosacral screw placement to obtain durable fixation and to prevent non-union.

Percutaneous technique using fluoroscopy or computer tomography [5,7,8] minimizes the rate of wound complications. However this technique requires a great experience of the surgeon ${ }^{[3]}$ and many $\mathrm{C}$-arm manipulation. Furthermore, digestive gases can make the radioscopic vision difficult.

This paper presents a computer assisted method. The main goals are to increase accuracy, thanks to the computer assistance, and to decrease the rate of wound complications, by using a percutaneous procedure. Our approach is based on computer assisted surgery using pre-operative CT-scan model registered with ultrasound data of the patient during surgery.

Clinical validation has started at the end of February 2000. Up to now four patients have been instrumented, three with two screws on one side of the pelvis, one with two screws on each side of the pelvic bone (because of arthrodesis of the two iliosacral joints). This clinical validation follows a cadavers study described in ${ }^{[1]}$. The major modification concerns the ultrasound acquisition. On cadavers, it was performed on the iliac crest, fossa iliaca lateral, ischiatic process and posterior side of the sacrum. To avoid problem of relative motion at fracture site, we are now working only on posterior part of the sacrum thanks to a smaller probe.

\section{Material and Methods}

Our protocol is divided into three steps.

\subsection{Pre-operative CT-Scan Segmentation and Surgical Planning}

A CT-scan acquisition of nearly 50 images with a $3 \mathrm{~mm}$ slice thickness is performed with a spiral CT GE Hispeed Advantage. The examination field includes the whole sacrum. A semi-automatic segmentation of the bone is performed in order to have a CT-based 3D model of the pelvis. 


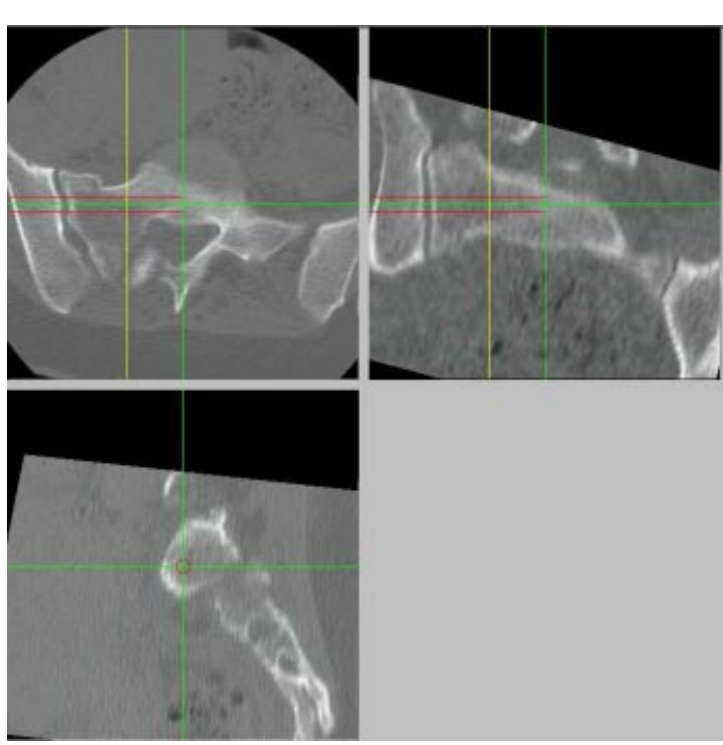

Fig. 2 : Surgical planning on CT-scan images.
Then, the surgeon defines the optimal placement of screws using an interactive interface that allows the computation of arbitrary planes orientation in the volume of CT-scan images (see Fig. 2). Each screw is described by an entry point, a direction, and a diameter. Two screw positions are defined : one from supraforaminal S1-S2 superior into the S1 vertebra's body, one from supraforaminal S1-S2 inferior into the S1 body. The screw tips are defined on the mid-sagittal line of the sacrum. The planning allows accurate determi-nation of each screw length

\subsection{Intra-operative Ultrasound Acquisition and Registration}

During surgery, a 6D optical localizer (Polaris, NDI - Toronto) is used. It locates the position and orientation of wireless customized rigid-bodies equipped with reflective markers (TIMC, laboratory). Rigid-bodies are fixed on tools used by the surgeon (see Fig. 3) : - a standard ultrasound probe (A) of 75 $\mathrm{MHz}$ frequency with an examination field of $2 \mathrm{~cm}$ width and $5 \mathrm{~cm}$ depth

- a linear tool (B) used for registration validity checking and drilling trajectory approach.

- a standard surgical power drill (C).

- a linear tool guide used during drilling and screwing (D).

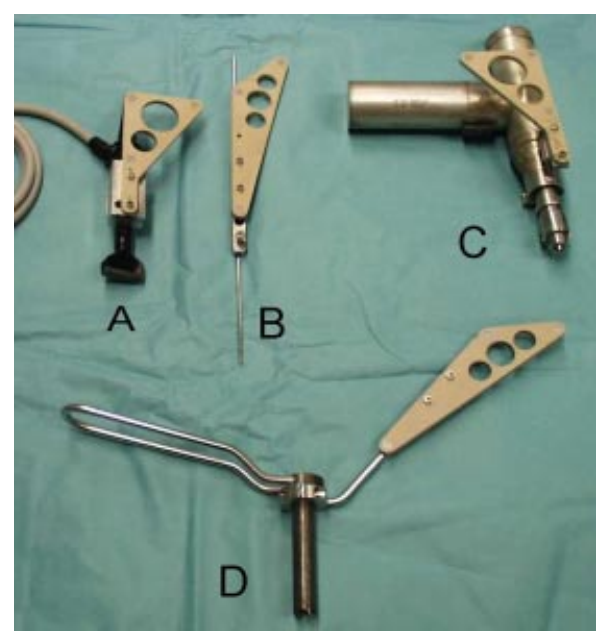

Fig. 3 : Tools used during surgery

A reference rigid-body is firmly fixed on the sacrum or in the posterior iliac crest on the opposite side of the fracture. It defines the intra-operative reference coordinate system that is used during the whole surgery. 
As described in ${ }^{[9]}$, we are using a standard ultrasound probe to image the bone and soft tissue interface of the sacrum (see Fig. 4). The patient is in prone position
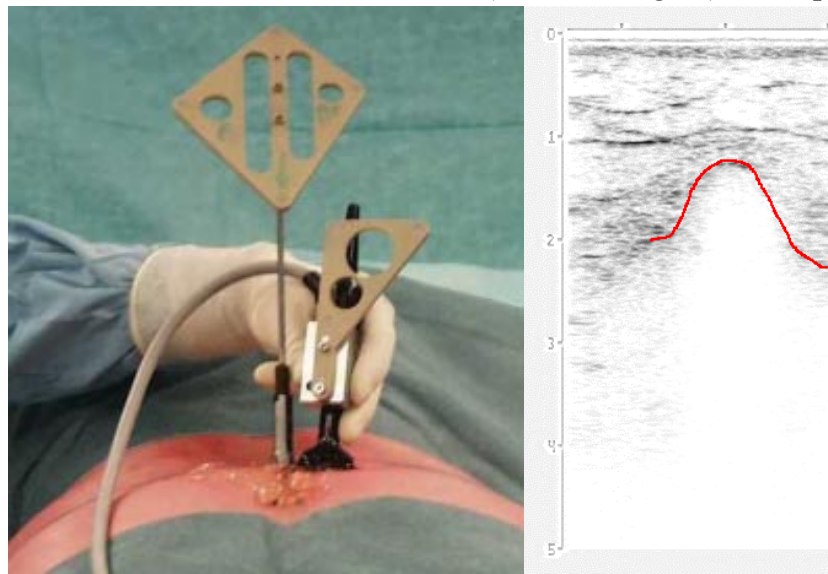

and a sterile transonic gel is used. Nearly 40 ultrasound images are acquired. Then, a segmen-tation procedure enables us to build 2D curves that belong to the bone surface on each image (see Fig. 5).

Fig. 4 : US acquisition.

Fig. 5 : US segmentation.

Using the 6D location and the calibration of ultrasound probe, those curves are converted into a cloud of 3D points referenced in the intra-operative coordinate system. These points are registered with the 3D CT-scan model of the pelvis (see Fig. 6) using surface based registration ${ }^{[14]}$. As a result, the optimal screw positions defined on the CT-scan are known in the intra-operative coordinate system. This technique avoid the insertion of feducial markers into the bones when constructing the pre-operative model.
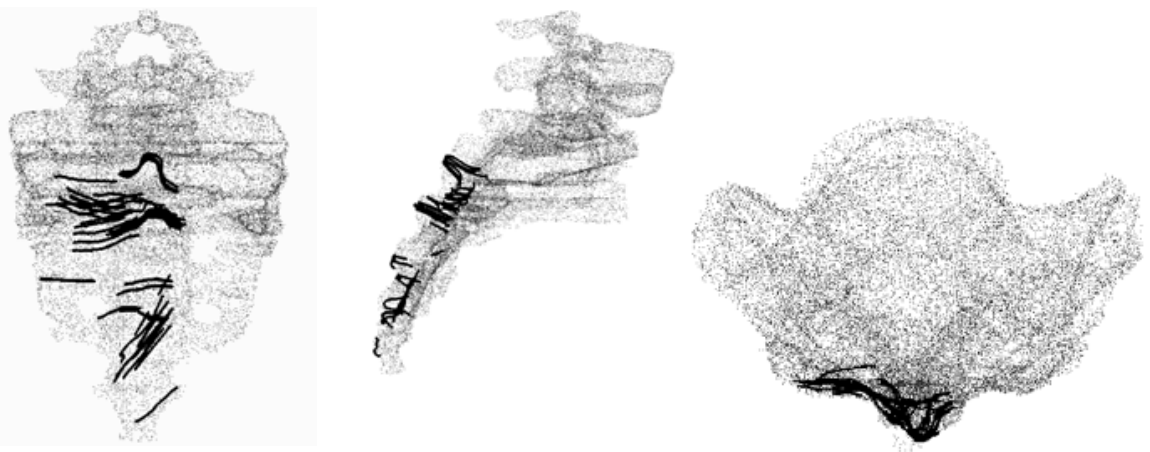

Fig. 6 : Registration between the CT-scan 3D model (in gray) and the 3D data obtained with the ultrasound segmentation (in black) . 


\subsection{Passive Drilling and Screwing Guidance}

The passive guidance process can then be started using a real-time navigation assistance. The first step consists in the registration validation. The surgeon uses the linear tool and put it on specific anatomical points. The tool axis and extremity are tracked and corresponding images are displayed. The surgeon is then able to check if displayed images really correspond to the tool position. Once the registration result is validated, the surgeon localizes the planned entry point and direction of the drilling trajectory (see Fig. 7).

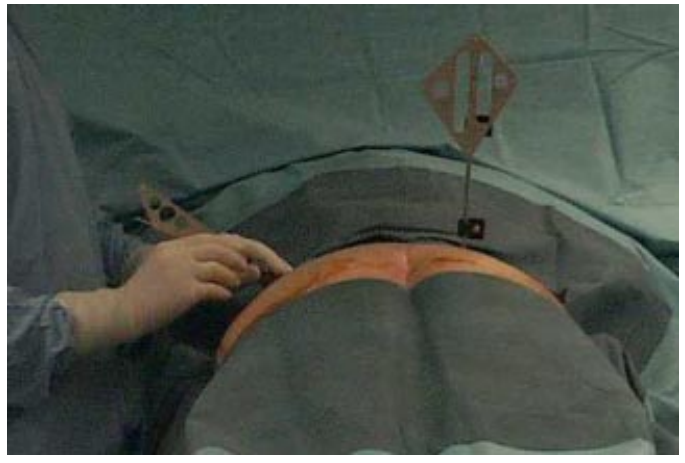

Fig. 7 : Navigation with the linear tool.

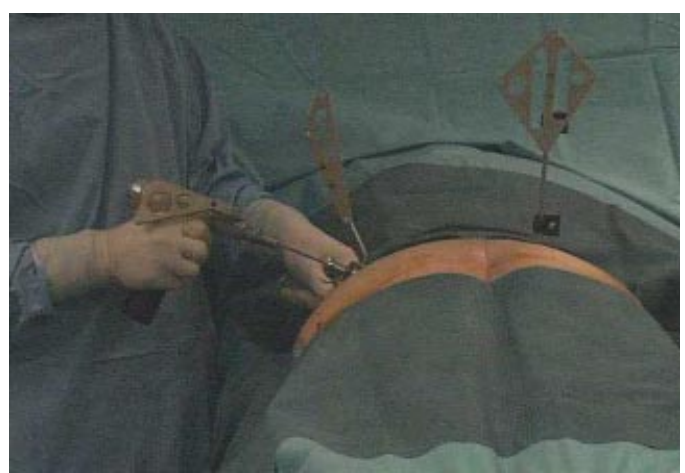

Fig. 8: Drilling Process.

The linear tool is positioned at the entry point of the bone and used to make a small hole. This step is done in order to find the trajectory position in a less invasive way than with the drill guide, that has a bigger diameter. Furthermore, this hole will be used to prevent unexpected motion of the drill tip on the bone at the beginning of the drilling process.

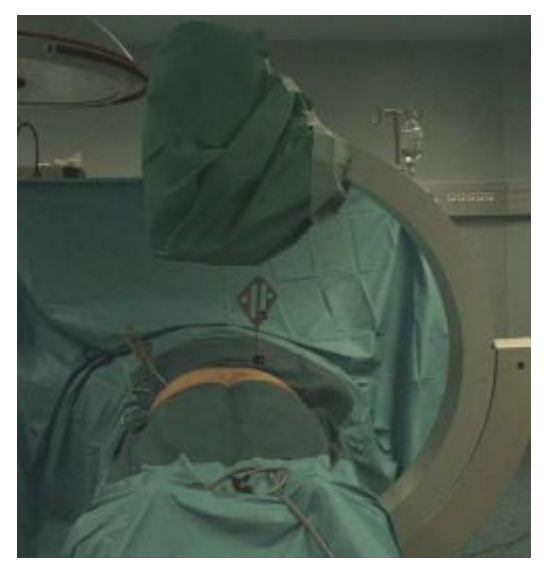

Fig. 9: X-ray checking.

The second step consists in finding the entry point with the drill guide. Like the linear tool, its axis and extremity have been calibrated. Once the entry point and direction of the trajectory have been found, the drilling process is started (see Fig.8). The power-drill calibration allows the monitoring of the drill depth penetration and the comparison with the maximum depth penetration allowed by the CT-scan planning. Before crossing the S1-S2 foramen, drill trajectory is checked thanks to one x-ray image (see Fig. 9) as proposed in ${ }^{[10]}$. 
At the end of the drilling process, the surgeon takes the power drill off but leaves the drill guide in the correct position. The third step is the screwing. Thanks to a tool device, the surgeon can insert the screw driver within the guide. As a consequence, the screw is guided into the hole whereas without assistance, it can be difficult and takes several minutes.

During each step, real-time tool tracking and re-sliced images are displayed with tool position shown on CT-scan images. In addition, a crosshair alignment system allows an easy comparison between the planned trajectory and the real position of the tool (see Fig. 10).
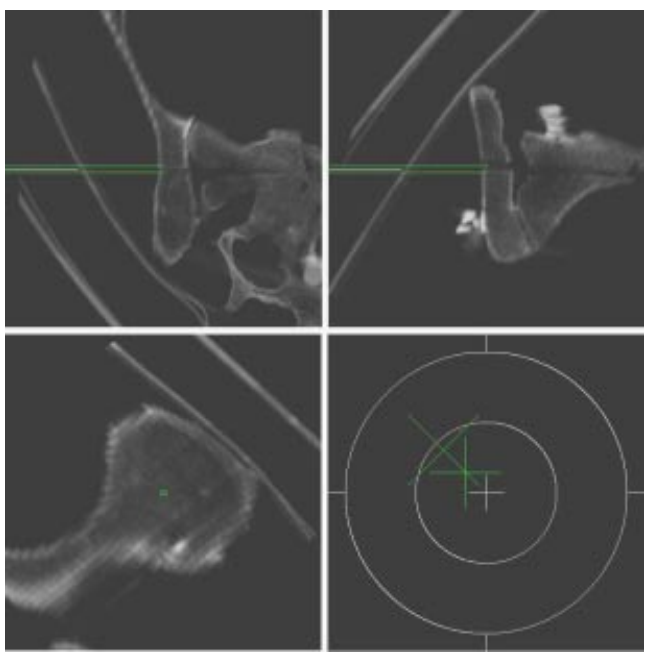

Fig. 10: Computer screen display

Thanks to this assisted guidance, $6.5 \mathrm{~mm}$ diameter screws for cancellous bones (Synthes inc, Etupe, France) are easily inserted in the pelvic bone.

\section{Clinical Validation}

Since the end of February, four pelvis have been instrumented with a total of 10 screws. X-ray exposition time during surgery is recorded. This value is important, and should decrease thanks to the computer assistance in comparison with percutaneous surgery under fluoroscopy. X-ray images are acquired to check the correct position of the drill and screw. Ultrasound acquisition, segmentation and registration time are also recorded. They are additional steps to a classical surgery and directly influence the surgical time. After surgery, a post-operative CT-scan is performed in planes positioned along and perpendicular to each screw axis by report to the topography of the lumbosacral trunk and the S1 root.

These images are used to take measurement on screw positions (see Fig. 11). $\mathrm{A}$ is the distance from the screw to the anterior cortex of the sacrum. B is the distance from the screw to the spinal canal or to the foramen. $\mathrm{C}$ is the distance from the tip of the screw to the mid-sagittal line of the sacrum. A and B values are measured if the screw is in the bone.

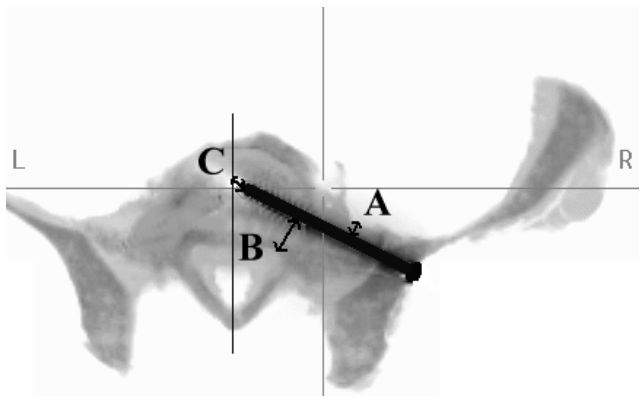

Fig. 11 : Method of screw position measurement. 
We have established a security score that depends on A and B values such as : If $\mathrm{A}$ or $\mathrm{B}$ equal 0 , security score is $0 \%$ because one anatomical limit is reached. If $\mathrm{A}=\mathrm{B}$, security score is $100 \%$ because the screw is accurately centered.

\begin{tabular}{|ll|c|c|c|c|}
\hline & & $\mathrm{A}(\mathrm{mm})$ & $\mathrm{B}(\mathrm{mm})$ & $\mathrm{C}(\mathrm{mm})$ & Score $(\%)$ \\
\hline Patient 1 & Sup & 10.3 & 3 & 0 & 45 \\
\cline { 3 - 6 } & Inf & 3.6 & 7.2 & 0 & 67 \\
\hline Patient 2 & Sup & 8 & 3.5 & 3.5 & 61 \\
\cline { 3 - 6 } & Inf & 5.5 & 2 & 5.5 & 53 \\
\hline Patient 2 & Sup & 3.5 & 4.5 & 4.6 & 88 \\
\cline { 3 - 6 } & Inf & 3 & 5.5 & 5 & 71 \\
\hline Patient 3 & Sup & 10.4 & 5.2 & 1.5 & 67 \\
& Inf & 8.7 & 4.3 & 0 & 66 \\
\hline Patient 4 & Sup & 0 & 4.5 & 0 & 0 \\
\cline { 3 - 6 } & Inf & 3 & 3 & 0 & 100 \\
\hline
\end{tabular}

Table 1 : Measurements.

Values reported in Table 1 show that all screws were in the correct position and preserve the close anatomical elements. Anatomical limit has been reached only one time

Average x-ray irradiation time measured during surgery has a value of 0.35 minutes and ultrasound acquisition, segmentation and registration has an average value of 24 minutes. All those values are recorded in an observation book in which blood loss, complications, prospective follow-up with neurological evaluation and self-evaluation of pain at 7 days, 45 days and 3 months are also reported. Current status of the validation shows that the four patients have an excellent neurological status.

\section{Discussion}

A study on percutaneous surgery under fluoroscopy has been made on 30 patients. Comparing those results with the ones obtained with the computer assisted technique allows us to draw table2. However, result tendencies have to be confirmed on a larger number of cases.

The first result of this table concerns the number of outside bone screws: $28 \%$ with classical technique and $0 \%$ with the computer assisted technique. On these outside bone screws, one over three has directly induce a neurological lesion. That represents $9.3 \%$ of screws inserted with the classical method.

With the computer assisted technique, the x-ray irradiation time has been divided by three. Moreover, this duration should decrease with the experience of the surgeon and its confidence in the system. 


\begin{tabular}{|c|c|c|}
\hline & Under fluoroscopy & Computer assisted \\
\hline Number of patients & 30 & 4 \\
\hline Number of screws & 43 & 10 \\
\hline $\begin{array}{l}\text { Number of outside bone } \\
\text { screws }\end{array}$ & $(28 \%)$ & $(0 \%)$ \\
\hline $\begin{array}{l}\text { Neurological lesion due to } \\
\text { screw }\end{array}$ & $(9.3 \%)$ & $(0 \%)$ \\
\hline $\begin{array}{l}\text { X-ray exposition time } \\
\text { (min) }\end{array}$ & $1.07 \mathrm{~min}$ & $0.35 \mathrm{~min}$ \\
\hline $\begin{array}{l}\text { Average security score } \\
(\%)\end{array}$ & $47.1 \%$ & $61.8 \%$ \\
\hline
\end{tabular}

Table 2 : Comparison between classical and computer assisted techniques.

The major drawback of our system is the time needed for ultrasound acquisition, segmentation and registration. The increase time of surgery was nearly 25 minutes. The ultrasound acquisition time will probably decrease with the surgeon's learning. Research is currently performed to develop an automatic segmentation and registration of ultrasound data with $3 \mathrm{D}$ model. This will decrease the time and the possible inaccuracy of ultrasound segmentation due to images quality.

Cadaver study has shown registration average root mean squares error of 1.5 degrees and $2.6 \mathrm{~mm}$. Cadaver study average security score was $52.8 \%$. We have obtained $61.8 \%$ for the clinical validation. This accuracy increasing may be due to ultrasound images quality that was bad on cadaver because of deshydratation.

\section{Conclusion}

In comparison with the percutaneous technique under fluoroscopy, the computer assisted technique allows the real-time navigation in CT-scan images and divide the $\mathrm{x}$-ray exposition by three. The ultrasound based registration with CT-scan model takes advantage on feducial markers registration ${ }^{[11,12]}$ and method used for spine surgery ${ }^{[13,14,15]}$ because of wound complications decreasing.

The percutaneous technique described in this paper is secure enough to implant 2 screws of $6.5 \mathrm{~mm}$ diameter through the iliosacral joint. The clinical validation has started at the end of February 2000 and 4 pelvic have already been successfully instrumented with 10 screws. Clinical validation with computer assisted technique is going on. 


\section{References}

1. Tonetti J, Carrat L, Lavallee S, Pittet L, Cinquin Ph, Merloz Ph and Chirossel JP. Percutaneous iliosacral screw placement using image guided techniques. Clin. Orthop. (354), p. 103-110, 1998.

2. Waddel JP, Pennal GF, Tile M and Garside H. Pelvic disruption : Assessment and classification. Clin. Orthop. (151), p. 12-21, 1978.

3. Freese J, Templeman D, Schmidt A and Weisman I. Proximity of iliosacral screws to neurovascular structures after internal fixation. Clin. Orthop. (329), p. 194-198, 1996.

4. Letournel E. Pelvic fractures. Rev. Chir. Orthop. (10), p 145-148, 1978.

5. Matta JM and Saucedo T. Internal fixation of pelvic fracture. Clin. Orthop. (242), p 83-97, 1998.

6. Tile M and Pennal GF. Pelvic disruption : Principe of management. Clin. Orthop. (151), p 56-64, 1980.

7. Xoombs RJ, Jackson WT, Ebraheim NA, Russin JJ and Holiday B. Percutaneous computer-tomography stabilization of pelvic fractures. Preliminary report. Journal Orthop. Trauma. (1), p 197-204, 1987.

8. Routt ML and Simonian PT, Closed reduction and percutaneous skeletal fixation of sacral fractures. Clin. Orthop. (329), p121-128, 1996.

9. Barbe C, Troccaz J, Mazier B and lavallee S. Using 2.5D echography in computer assisted spine surgery. Engineering in Medicine and Biology Society Proceedings. San Diego, Institute of Electrical and Electronics Engineers Inc p 160-161, 1993.

10. Routt ML, Simonian PT, Agnew SG and Mann FA. Radiographic recognition of the sacral ala slope for optimal placement of iliosacral screws: a cadaveric and clinical study. J Orhtop. Trauma.(10:3),p 171-173, 1996.

11. Jacob AL. Computer assistance in pelvic and acetabular fractures. In L. Nolte editor, CAOS 96, Bern (CH), 1996. Muller Institute.

12. Hu R, Glossop N, Steven D and Randle J. Accuracy of image guided placement of iliosacral lag screws. In Troccaz J and al. Editors, CVRMed-MRCAS'97 Proc., LNCS Series 1205, p 593-596, Springer, 1997.

13. Lavallee S, Sautot P, Troccaz J, Cinquin P and Merloz P. Computer Assisted Spine Surgery : a technique for accurate transpedicular screw fixation using CT data and a $3 \mathrm{D}$ optical localizer. Journ. Of Image guided Surgery (1), p 65-73, 1995.

14. Lavallee S, Szeliski R and Brunie L. Anatomy-based registration of 3D medical images, range images, X-ray projections, 3D models using Octree-Splines. In Taylor R, Lavallee S, Burdea G and Mosges R, editors, Computer Integrated Surgery, p 115-143, MIT Press, Cambridge, MA, 1996.

15. Merloz P, Tonetti J, Eid A, Faure C, Lavallee S, Troccaz J, Sautot P, Hamadeh A and Cinquin P. Computer assisted spine surgery. Clin. Orthop. And Related Research (337) p 86-96, 1997. 